\title{
A PROPOSED SUPERCONDUCTING PHOTOEMISSION SOURCE OF HIGH BRIGHTNESS
}

\author{
H.Chaloupka, H.Heinrichs, H.Piel \\ Berglsche Universität-Gesamthochschule Wuppertal \\ C.K. Sinclalr \\ CEBAF, Newport News, Virginia, USA \\ F.Ebeling, T. Welland \\ DESY, Hamburg \\ U.Kleln, H.P.Vogel \\ Interatom GmbH., Bensberg
}

Abstract: Short wavelength free-electron laser oscillators require electron beams of high brightness, and, in some application, high average power. We describe the design of an electron Injector for the production of a bunched $\mathrm{cw}$ electron beam of high brightness, low energy spread, and potentially high beam power. It consists of a superconducting reentrant cavity housing a photoemission cathode which is irradiated with short light pulses of a mode-locked frequency doubled Nd:YAG laser. The experimental layout of the "superconducting photoemission source" is described together with its components: the photocathode preparation chamber, the cavity, the cryogenic setup, and the beam analysis system. The conceptual beam parameters are discussed and first results of an emittance calculation using a particle in cell computer code are given.

\section{Introduction}

High brightness electron beams are required for a number of accelerator applications as well as for short wavelength free-electron laser (FEL) oscillators. Photocathodes Irradiated by $\mathrm{cw}$ or pulsed lasers are sources of very bright, high current density electron beams. Electron injectors based on photoemitter technology are presently under development at several laboratories [1,2]. FEL's operating in the XUV and soft $x$-ray wavelength range require low energy spread, long macropulse duration, and high average power as well as high beam brightness [3]. Superconducting of linacs including a superconducting injector are the favoured candidate technology for these applications. Due to the high $Q$ factor these linacs can be operated even in a cw mode in which a high peak current bunch is placed in every rf bucket. Furthermore, the high $Q$ factor opens the possibility of energy recovery by recycling the beam through the linac [4].

Superconducting cavities for accelerator application have been extensively developed in the last years [5]. Peak surface electric fields of more than $30 \mathrm{MV} / \mathrm{m}$ can be reached reliably and maintained continuously.

\section{Conceptual Parameters and Experimental Setup}

Based on the present achievements with superconducting cavities, photoemission cathodes, and laser systems, it is reasonable to design an electron injector with the parameters of Table 1. Different columns in this table give the performance anticipated with different laser systems. The cavity and the photocathode are the same in all of these versions.

Table 1

Conceptual electron beam parameters

\begin{tabular}{llccc}
\hline Beam kinetic energy & {$[\mathrm{MeV}]$} & 1.3 & 1.3 & 1.3 \\
Peak current & {$[\mathrm{A}]$} & 2.3 & 30 & 200 \\
Charge per bunch & {$[\mathrm{nC}]$} & 0.16 & 0.15 & 14 \\
Pulse length & {$[\mathrm{ps}]$} & 70 & 5 & 70 \\
Repetition rate & {$[\mathrm{Hz}]$} & $125 \cdot 10^{6}$ & $125 \cdot 10^{6}$ & 100 \\
\hline
\end{tabular}

The construction of the actual electron source will follow the development of a good quantum efficiency photocathode on a niobium surface. Various cholces for the laser system will permit many operational modes, from $\mathrm{cw}$ beam with relatively low peak current, through pulsed operation with various combinations of duty factor and peak current, to a "burst" mode with very high peak current bursts composed of a small number of micropulses.

Fig. 1 shows a schematic view of the experimental setup for the electron injector and its beam diagnostic system. The superconducting cavity, which is of reentrant type, is shown in more detall in Fig. 2.

It is mounted in a vertical bath cryostat, as this arrangement is both easier to operate and much less expensive than a horizontal cryostat. The photocathode is prepared in a chamber on top of the cryostat. After preparation, the cathode is transferred under vacuum into the cavity. Due to the low rf losses of the cavity wall the cathode is connected to it via a band rejection filter.

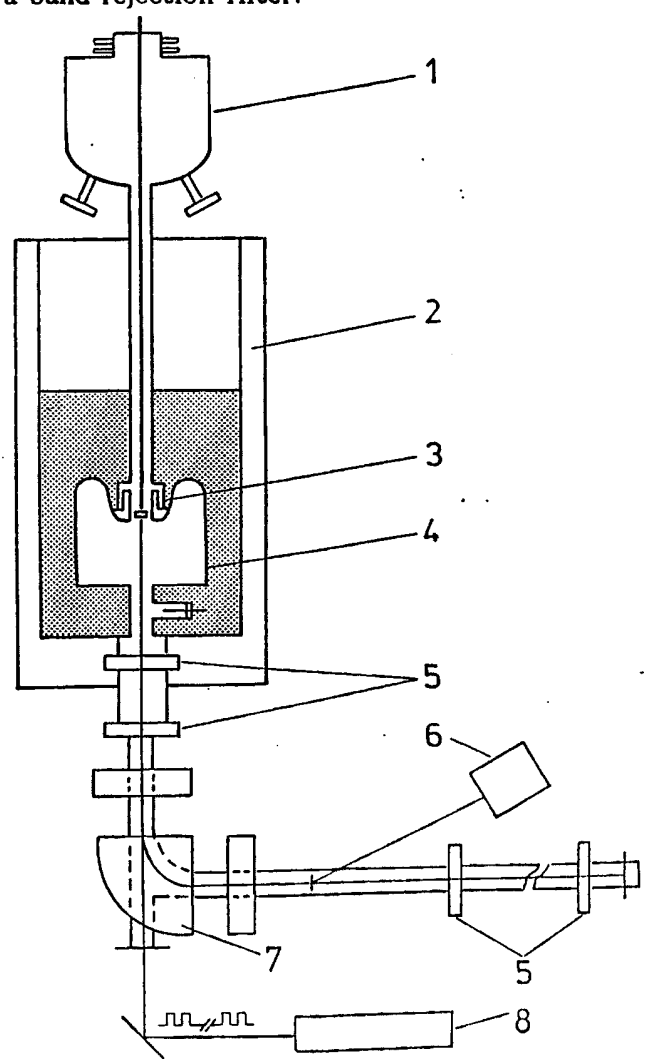

Fig. 1: Experimental setup of the injector

1: photocathode preparation chamber,

2: bath cryostat, 3: photocathode, 4: reentrant cavity,

5: wire scanner monitor, 6 : streak camera,

7: spectrometer, 8: Nd:YAG laser 


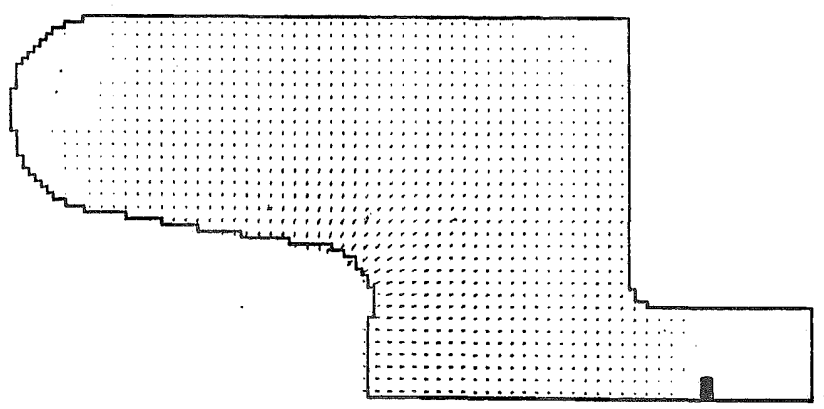

Fig. 2: Superconducting reentrant cavity

The laser beam enters the cavity through the beam tube from the bottom of the cryostat and Illuminates the cathode. The electron beam is accelerated in the gap of the reentrant cavity, Its emittance can be measured with wire scanner monitors below the cryostat. Then the beam is bent isochronously in a magnetic spectrometer to measure its energy before it passes a thin fused silica screen to create Cerenkov radiation. This can be analysed with a streak camera. The charge of the beam is measured with a Faraday cup at the end of the beam line.

\section{Components}

The cathode preparation system

The most extensively developed cathodes are gallum arsenide (GaAs) and cesium antimonide $\left(\mathrm{Cs}_{3} \mathrm{Sb}\right)$. The latter is easier to fabricate than the GaAs photocathode, and is rather more resistant to degradation by residual gases. The $\mathrm{Cs}_{3} \mathrm{Sb}$ photocathode has higher optical absorption than GaAs, and is typically thinner. As a consequence, it is able to produce shorter electron pulses than GaAs. It has, however, a considerably lower quantum efficiency than GaAs, which places greater demands on the optical source. If there are no limitations from space charge, field strength, and available charge, the current delivered by a photocathode

$$
\mathrm{I}=\mathrm{P} \cdot \mathrm{QE} / \mathrm{Eph}
$$

where $I$ is the photocurrent in Ampere, $P$ is the laser power in watts, $Q E$ is the photocathode quantum efficiency, and Eph is the photon energy in electron valts.

The development work on cesium antimonide photocathodes has been done by Los Alamos National Laboratory and the Thermo Electron Corporation. In a series of developments over several years, these two groups have demonstrated both high current capability and high brightness in electron guns. An of gun operating at $1300 \mathrm{MHz}$ has reported currents up to 390 Ampere from a $1 \mathrm{~cm}^{2}$ cathode mounted in the $\mathrm{rf}$ cavity wall, and has reported in addition beam brightness figures between 1.4 and $9 \cdot 10^{10} \mathrm{~A} /(\mathrm{m}-\mathrm{rad})^{2}$. The largest single pulse charge reported by the Los Alamos group was $27 \mathrm{nC}$. There seems to be no reason why such photocathodes cannot deliver beams of 1 to $14 \mathrm{nC}$ per bunch at bunch repetition rates likely limited by the avallable $\mathrm{rf}$ power and with beam brightness of $10^{11} \mathrm{~A} /(\mathrm{m}-\mathrm{rad})^{2}$ or greater.

The photocathode preparation chamber is shown schematically in Fig. 3. The system is very similar to that developed at SLAC for the study of III-V semiconductor photocathodes [6]. It will be used to explore a variety of photocathode preparation techniques, primarily for the alkali antimonides. Initial work will be done with cesium antimonide but we anticipate working also with higher quantum efficiency cathodes, such as sodium potassium antimonide.

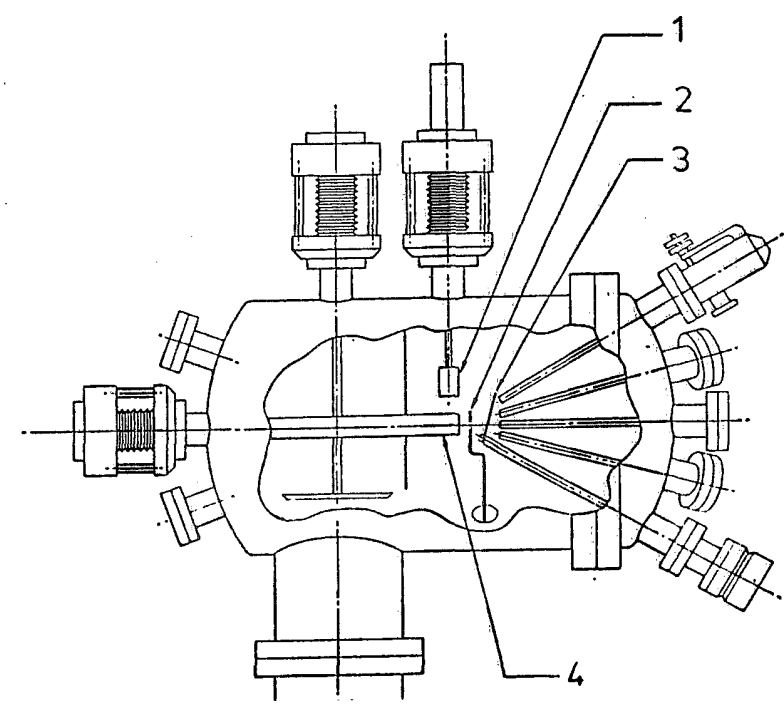

Fig. 3: Photocathode preparation chamber

1: quartz microbalance, 2: collector ring, 3: shutter, 4: cathode stem.

The cathodes are formed on the small area flat face of the cathode stem. This face will be niobium. The cathodes are formed by deposition of the appropriate materials on the face of the stem. One of the interesting questions will be whether the cathode material will become superconducting due to the proximity effect. This would reduce heating of the cathode by rf losses.

The operational lifetime of the cathode is a matter of great importance. The mechanisms presently believed respons ible for photocathode degradation in actively pumped UHV chambers are (1) poisoning by various residual gases, (2) damage by ton bombardement, and (3) alkali desorption. All these mechnisms should be absent in the low temperature environment of a superconducting cavity.

The techniques developed in this system for optimal photocathode preparation on niobium will be transferred to a much smaller system mounted on top of the injector cryostat for the ultimate fabrication.

The laser system

The characteristics of the cathode will determine the demands made upon the laser system. The only reasonable (and currently the only commercially available) laser system which is capable of producing $\mathrm{c} w$ trains of picosecond pulses is a cW mode-locked, frequency doubled Nd:YAG or Nd:YLF laser. At the wavelength of a frequency doubled Nd:YAG laser, i.e. $532 \mathrm{~nm}$, the best cesium antimonide photocathodes have a quantum efficiency of somewhat over 4\%. According to Eq. (1) it is possible to produce an average current of $20 \mathrm{~mA}$ if the average laser power is 1 watt. For economic reasons, our test system will not be equipped with a high power klystron. Therefore the duty factor has to be restricted to about $2 \%$.

Typically, mode-locked laser systems produce a pulse repetition rate between 70 and $160 \mathrm{MHz}$. For a cavity frequency of $500 \mathrm{MHz}$, the laser may be conveniently operated at the fourth or eighth subharmonic of this frequency, or the laser beam may be optically multiplexed to $500 \mathrm{MHz}$. For the example above one gets in case of operation at the fourth subharmonic a peak current of $2.3 \mathrm{~A}$, respectively $0.16 \mathrm{nC}$ per bunch.

In a frequency doubled mode-locked Nd:YAG laser the pulse width is about 70 ps or less. These pulses may be 
shortened by the use of YLF as the lasing medium, and by the use of intra-cavity etalongs to effectively broaden the laser cavity bandwidth. Pulse width of 5 to 10 ps should be obtained by these methods. For a $500 \mathrm{MHz}$ cavity, these pulses occupy between 1 and 2 degree of RF phase, which produce a narrow energy spread. The amount of charge that can be removed in such short time from photocathode and the degradation of energy spread in such a pulse has to be measured. Guide lines are given by the Los Alamos results. There $1 / 6$ of the available charge, as given by Gauss's law was removed in a 60 ps pulse and no bunch lengthening was observed.

None of the commercially avallable laser systems has the possibility to be tuned and frequency locked to an external standard, e.g. to superconducting cavity which is a good frequency standard. Problems may arise from instabilities in light intensity and from a jitter of the light pulses in time.

To operate the system in a high current density regime with large pulse charge and low duty factor, it will be necessary to amplify the laser output for shorter periods of time. This can be done by chopping the laser output with a pockels cell to produce microsecond duration bursts of mode-locked pulse trains at repetition rate of 10 to $100 \mathrm{~Hz}$. These pulse trains are then amplifying with a laser amplifier. Peak-currents of more than $200 \mathrm{~A}$ are expected.

\section{The superconducting cavity}

Due to the high $Q$ factor of the superconducting cavity the main advantage of it is the high fleld strength which can be reached and maintained continuously. It is not to be optimized for high shunt impedance, but rather to achieve high surface electric fields at the cathode and high accelerating voltage. In addition, the cavity shape and fields must be optimized to minimize emittance growth. Detalled particle dynamics simulation must be carried out to achieve these optimizations. The reentrant cavity shaped is well suited to obtain high cathode fields, and offers the advantage of relatively small size even at $500 \mathrm{MHz}$ frequency. In an early stage of the injector program the cavicy will be built to study its field limitations under special consideration of electron multipacting, normal conducting defects and field emission.

Parallel to this several possibilities of mounting the photocathode in the wall of the cavity will be tested. Specifically a band rejection filter will be designed and tested in a copper model of the cavity.

The beam diagnostic system

An important aspect of understanding the dynamics of photo emission and the electron beam in the injector is the ability to perform time-resolved diagnostics with the appropriate resolution. The relevant time structure in cw operation is established by the 5 resp. 70 ps long micropulses seperated 2 resp. $8 \mathrm{~ns}$. Due to the low average microwave drive power available, the beam must be chopped into macropulses of 1 to 100 us duration. The large range of time scales involved can only be analysed with sufficient precision with a streak camera. Following measurement of the beam divergence and emittance with wire scanner monitors, the beam strikes a thin fused sllica Cerenkov radiator. This provides a visible light signal which accurately follows the time structure of the beam. The light is analysed with a time resolution of 3 ps by a commercial streak camera.

Beam dynamics

The dynamics of the partice motion are simulated with the partice-in-cell code TBCI-SF [7]. The code starts with the cavity fields calculated by URMEL. The code simulates the movement of the electron bunch through the cavity taking into account the space charge forces acting on the bunch. In a series of calculations the cavity shape will be optimized with respect to a reduction of emittance growth. The first calculations were carried out for the preliminary design shown in Fig. 2. Assuming a surface field at the cathode of $16 \mathrm{MV} / \mathrm{m}$ a bunch generated from a 10 ps laser pulse carrying $0.157 \mathrm{nC}$ is accelerated to an energy of $1.8 \mathrm{MeV}$ with an energy spread of $0.25 \%$. When it leaves the cavity at the far end of the cutoff tube it has a length of $2.6 \mathrm{~mm}$ and a transverse emittance of $60 \pi \mathrm{mm}$ mrad. With small changes of the cavity shape it was possible in a second step to reduce emittance to $45 \pi \mathrm{mm} \mathrm{mrad}$. There is good chance to reduce this value further and to increase the performance of the injector.

\section{Acknowledgement}

This work is funded in part by the Minister for Science and Research of the State of NorthRhine-Westfalia.

\section{References}

[1] P.E. Oettinger, R.E. Shefer, D.L. Birx, M.C. Green Photoelectron Sources: Selection and Analysis, in Proceedings of the 9th International FEL. Conference. Will amsburg (to be published)

[2] R.L.Sheffield, "High Brightness Electron Injectors", in Proceedings of the ICFA Workshop on Low Emittance Beams, Brookhaven National Laboratory, March 1987, BNL 52090.

[3] John C. Goldstein, "Electron Beam Requirements for Soft X-Ray/XUV Free-Electron-Lasers", ibid. Ref.[2].

[4] T.I. Smith, H.A. Schwettman, R. Rohatgi, Y. Lapierre and J. Edighoffer, "Development of the SCA / FEL for Use in Biomedial and Materials Sciences Experiments", Nucl. Instr. Meth. A259, pp 1-7, 1987.

[5] K. Shepard, editor, Proceedings of the Third Workshop on RF Superconductivity, ANL-PHY 88-1, Vol. I.

[6] C.K. Sinclair, "The SLAC Lasertron Project", in Proceedings of the Symposium on Advanced Accelerator Concepts, Madison, Wisconsin, Aug. 1986, AIP Conference Proceedings, vol. 156.

[7] F. Ebeling, P. Schuitt and T. Weiland, "Selfconsistent Simulation of High Power Tubes with TBCI-SF", these proceedings 